\title{
Relationship of frailty with treatment modality selection in patients with muscle-invasive bladder cancer (FRART-BC study)
}

\author{
Hiromichi Iwamura ${ }^{1}$, Shingo Hatakeyama ${ }^{2}$, Masaki Momota ${ }^{1}$, Yuta Kojima ${ }^{1}$, Takuma Narita ${ }^{1}$, \\ Teppei Okamoto ${ }^{1}$, Naoki Fujita ${ }^{1}$, Itsuto Hamano ${ }^{1}$, Kyou Togashi ${ }^{1}$, Tomoko Hamaya ${ }^{1}$, Tohru Yoneyama ${ }^{3}$, \\ Hayato Yamamoto ${ }^{1}$, Takahiro Yoneyama ${ }^{3}$, Yasuhiro Hashimoto ${ }^{1}$, Chikara Ohyama ${ }^{1,2,3}$
}

${ }^{1}$ Department of Urology, Hirosaki University, Graduate School of Medicine, Hirosaki, Japan; ${ }^{2}$ Department of Advanced Blood Purification Therapy, Hirosaki University, Graduate School of Medicine, Hirosaki, Japan; ${ }^{3}$ Department of Advanced Transplant and Regenerative Medicine, Hirosaki University, Graduate School of Medicine, Hirosaki, Japan

Contributions: (I) Conception and design: S Hatakeyama, C Ohyama; (II) Administrative support: None; (III) Provision of study materials or patients: None; (IV) Collection and assembly of data: M Momota, Y Kojima, T Narita, T Okamoto, N Fujita, I Hamano, K Togashi, T Hamaya; (V) Data analysis and interpretation: H Iwamura, S Hatakeyama, T Yoneyama, H Yamamoto, T Yoneyama, Y Hashimoto, C Ohyama; (VI) Manuscript writing: All authors; (VII) Final approval of manuscript: All authors.

Correspondence to: Shingo Hatakeyama, MD. Department of Advanced Blood Purification Therapy, Hirosaki University Graduate School of Medicine, 5 Zaifu-chou, Hirosaki 036-8562, Japan. Email: shingoh@hirosaki-u.ac.jp.

Background: We aimed to investigate the association of frailty with treatment selection in patients with muscle-invasive bladder cancer (MIBC) as frailty is one of the key factors for modality selection.

Methods: We retrospectively evaluated frailty in 169 patients with MIBC from January 2014 to September 2020 using the Fried phenotype, modified frailty index, and frailty discriminant score. The primary purpose was comparing the frailty between the patients who underwent radical cystectomy (RC) with those who had trimodal therapy (TMT) for bladder preservation. Secondary purposes were comparing the frailty between the groups and the effect of TMT on overall survival adjusting the frailty by multivariate Cox proportional hazards analysis using inverse probability of treatment weighting (IPTW)-adjusted model.

Results: Of 169 patients, 96 and 73 were classified into the RC and the TMT groups, respectively. The median age of the TMT group was significantly higher than that of the RC group (80 vs. 69 years). Frailty levels and prevalence in the Fried phenotype, modified frailty index, and frailty discriminant score were significantly higher in the TMT group than those in the RC group. Logistic regression analysis showed that frailty was significantly associated with the TMT selection. Overall survival was significantly shorter in the TMT group by the IPTW-adjusted Cox regression analysis (hazard ratio 2.48, P=0.043).

Conclusions: Frailty was significantly different between the RC and TMT in patients with MIBC and might be one of the key factors for treatment selection.

Keywords: frailty; muscle-invasive bladder cancer (MIBC); cystectomy; trimodal therapy (TMT); bladder preservation

Submitted Oct 21, 2020. Accepted for publication Jan 18, 2021.

doi: $10.21037 /$ tau-20-1351

View this article at: http://dx.doi.org/10.21037/tau-20-1351

\section{Introduction}

Radical cystectomy (RC) with urinary diversion is the standard treatment for nonmetastatic, muscle-invasive bladder cancer (MIBC) (1-5). However, not all patients are eligible for RC because patients with MIBC have the highest age among all cancers (6). A treatment option for patients who cannot undergo RC is trimodal therapy (TMT), which involves maximal transurethral resection of the bladder tumor (TURBT) followed by concurrent chemotherapy and radiation therapy. Recent studies 
demonstrated comparable survival outcomes when patients were appropriately selected (7-13). However, no level 1 evidence supported the definitive selection criteria for TMT. Hence, RC or TMT is selected based on the clinicalpathological characteristics, general status, and preference of patients under selection biases. Of those, frailty potentially plays a key role as frail patients are unfit for a highly invasive treatment modality in several urological malignancies (14-18). In the aging population, a treatment strategy for MIBC using frailty to support the experiencebased management needs to be established (19). However, no study has assessed the effect of frailty on treatment selection in patients with MIBC. We aimed to investigate the association of FRAilty with treatment selection (RC or TMT) in patients with muscle-invasive Bladder Cancer (FRART-BC study). We present the following article in accordance with the STROBE reporting checklist (available at http://dx.doi.org/10.21037/tau-20-1351).

\section{Methods}

\section{Patient selection and treatment protocols}

The patients included in this study were selected from our prospective study that evaluated the effect of frailty on outcomes in 1,003 patients with urological cancers $(20,21)$. Of 1,003 , this study evaluated 169 patients with MIBC (cT $\leq 4 \mathrm{a}, \mathrm{cNany}, \mathrm{cM} 0)$ treated with $\mathrm{RC}$ or TMT at the Hirosaki University Hospital between January 2014 and September 2020 as a post-hoc study. Patients with missing data were excluded. Of 169, 96 and 73 patients were classified into the RC and TMT groups, respectively. A treatment was selected by multiple urologists as a part of multidisciplinary board. All patients in the RC group received 2-3 cycles of neoadjuvant chemotherapy that included a platinum-based combination regimen, with either gemcitabine plus cisplatin or gemcitabine plus carboplatin, followed by RC, urinary diversion, and standard pelvic lymph node dissection (22). Surgical procedures were performed using the basic technique described previously $(23,24)$. In contrast, TMT included a safe complete TURBT, followed by a 50-60 Gy external beam radiotherapy to the bladder and pelvic lymph nodes with four simultaneous cycles of platinum-based systemic chemotherapy (gemcitabine plus cisplatin or gemcitabine plus carboplatin). Each patient received computed tomography and urine cytology every 3-6 months for follow-up after completion of treatment. Lost to follow-up was defined as censored.

\section{Frailty assessment}

All the patients underwent frailty assessment before treatment selection. The results of frailty test were blinded to the surgeons and radiologists until the final decision. Three frailty assessment tools were used, namely, Fried phenotype (FP), modified frailty index (mFI), and frailty discriminant score (FDS). The FP was based on five factors, including shrinking, handgrip strength, gait speed, exhaustion, and reduced physical activity (score 0-5) (25). The $\mathrm{mFI}$ was evaluated based on 11 factors, including cardiovascular, pulmonary, and neurogenic comorbidities (score 0-11) (26). The FDS is a comprehensive geriatric assessment tool for frailty including age, sex, physical status, mental status, and blood test results (hemoglobin, serum albumin, and renal function) $(20,21,27)$. The FDS was obtained using the following formula: $[6.8698+$ age $\times 0.0053+\operatorname{sex}($ male $=1) \times 1.4794+$ body mass index $\times$ $0.0105+$ handgrip strength $\times-0.0209+$ gait speed $\times 0.1993$ + exhaustion $(0$ or 1$) \times 0.0876+$ depression $(0$ or 1$) \times 0.2005$ + albumin $\times-0.9037+$ estimated glomerular filtration rate $\times$ $-0.0112+$ hemoglobin $\times-0.2868]$. Frailty was defined using cutoff values of frailty in $\mathrm{FP}(\geq 3), \mathrm{mFI}(\geq 2)$, and FDS $(\geq 2.30)$.

\section{Outcomes}

The primary objective of this study was comparing the frailty between patients in the RC group and those in the TMT group. Secondary purpose was evaluating the effect of TMT on overall survival (OS) adjusting the frailty using inverse probability of treatment weighting (IPTW)-adjusted Cox regression analysis (28). Exploratory objective was to evaluate the effect of TMT on OS among the non-frail patients.

\section{Statistical analysis}

Statistical analyses were performed using the bell curve in excel (Social survey research information Co., Ltd., Tokyo, Japan), GraphPad Prism 7.00 (GraphPad software, San Diego, CA, USA), and R 3.3.2 (the R foundation for statistical computing, Vienna, Austria). Categorical variables were compared using the Fisher's exact test or the $\chi^{2}$ test, whereas quantitative variables were expressed as median with an interquartile range. The differences between the groups were compared using the Student's $t$-test or the 
Table 1 Baseline characteristics of the participants

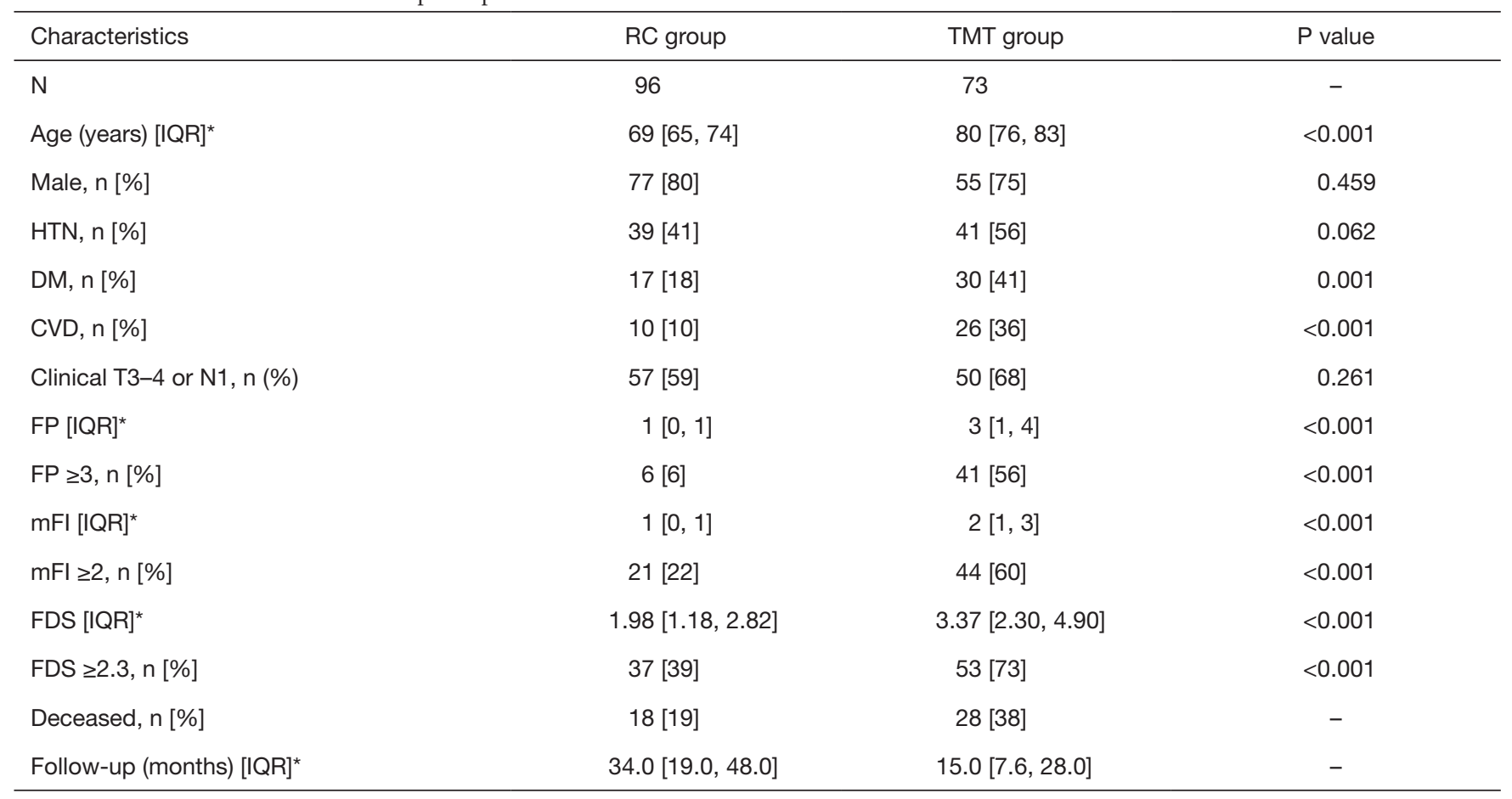

*, quantitative variables were expressed as median [IQR]. RC, radical cystectomy; TMT, trimodal therapy; IQR, interquartile range; HTN, hypertension; DM, diabetes mellitus; CVD, cardiovascular disease; FP, Fried phenotype; mFI, modified frailty index; FDS, frailty discriminant score.

Mann-Whitney U test. The optimal cutoff value was defined using the receiver operating characteristic (ROC) curve and the area under the ROC curve (AUC) value. Age, sex, and high-risk status (cT3-4/cN+) adjusted multivariate logistic regression analyses were performed to determine the effect of frailty on treatment selection. Odds ratio (OR) with $95 \%$ confidential interval (95\% CI) were obtained. OS of the RC and TMT groups were compared using the Kaplan-Meier method, and hazard ratio (HR) with $95 \%$ CI were obtained from the multivariate Cox proportional hazards analysis using IPTW-adjusted model. The propensity score-based IPTW method creates a pseudo-population and removes the background unbalances between the two groups and obtain unbiased estimates of average treatment effects (28). IPTWadjusted Cox regression analysis included propensity score calculation for treatment, calculation of inverse-probability weighting, and Cox regression analysis with the robust adjustment. The first step is the calculation of propensity score for the TMT group including high-risk disease (cT3-4/ $\mathrm{cN}_{+}$), and frailty using logistic regression analysis. The second step is the calculation of inverse-probability weighting for the treatment by the inverse of the probability of the "given" exposure. The weights of treatment are calculated using the formula: 1/propensity score for the RC group and $1 /(1$ - propensity score) for the TMT group. The third step is the multivariable Cox regression analysis with the robust adjustment including the two factors: treatment modality (the TMT group $=1$ ) and weights for the treatment. $\mathrm{P}$ values $<0.05$ were considered statistically significant.

\section{Ethics statement}

The study was conducted in accordance with the Declaration of Helsinki (as revised in 2013). The study was approved by the ethics review board of Hirosaki University School of Medicine (NO.: 2014-297) and individual consent for this retrospective analysis was waived.

\section{Results}

\section{Baseline characteristics of the participants}

Table 1 shows the characteristics of the study participants. The median age of the TMT group was significantly 
A

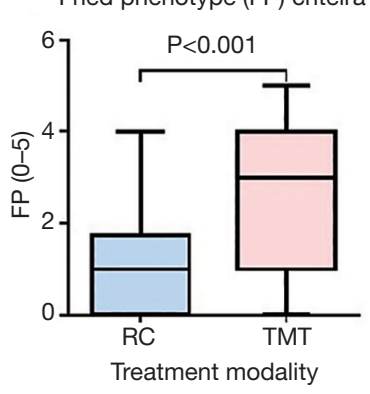

D

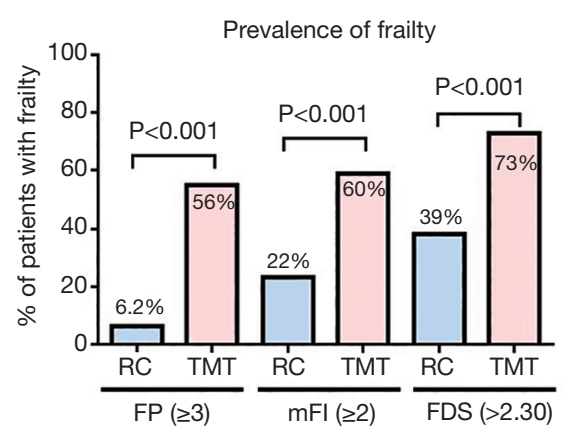

B

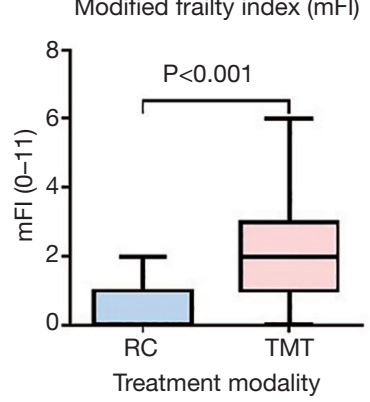

$E$

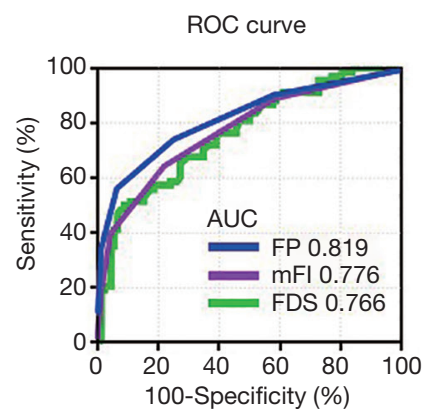

C Frailty discriminant score (FDS)

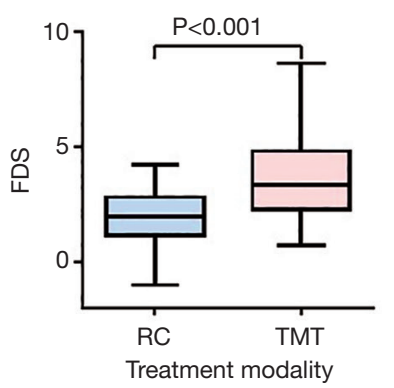

$\mathrm{F}$

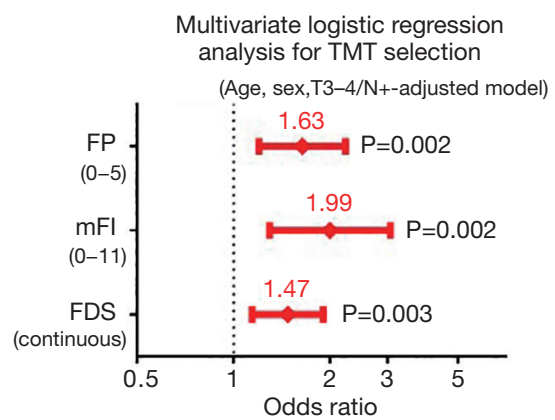

Figure 1 Primary outcomes: comparison of frailty between the RC and TMT groups. The FP (A), mFI (B), and FDS (C) scores were compared between the RC and TMT groups. (D) Prevalence of frailty in FP, mFI, and FDS were compared between the TMT and RC groups. (E) The area under the curve of FP, $\mathrm{mFI}$, and FDS between the RC and TMT groups were evaluated using the receiver operating characteristic curve. (F) Age, sex, and T3-4/N+-adjusted odds ratios of frailty on TMT selection logistic regression analyses for TMT selection was shown. RC, radical cystectomy; TMT, trimodal therapy; ROC, receiver operating characteristic.

higher than that of the RC group (80 vs. 69 years, $\mathrm{P}<0.001$ ). Moreover, the rates of comorbidities, such as hypertension (HTN), diabetes mellitus (DM) and cardiovascular disease (CVD), were significantly higher in the TMT group than in the RC group ( $56 \%$ vs. $41 \%$, $41 \%$ vs. $18 \%$, and $36 \%$ vs. $10 \%$, respectively). The TMT group showed a higher number of deceased patients than the RC group (38\% vs. 19\%). Sex, and high-risk disease (cT3-4/N+) were not significantly different between the groups.

\section{Primary outcomes: comparison of frailty between the $R C$ and TMT groups}

Figure 1 presents the frailty comparison between the RC and TMT groups. The FP, mFI, and FDS scores were significantly higher in the TMT group than in the RC group (1 vs. 3, 1 vs. 2, and 1.98 vs. 3.37, respectively, $\mathrm{P}<0.001$, Figure $1 A, B, C)$. Frailty prevalence was significantly higher in the TMT group than in the RC group (FP, $56 \%$ vs. $6.2 \%$; mFI, $60 \%$ vs. $22 \%$; and FDS, $73 \%$ vs. $39 \%$, respectively; $\mathrm{P}<0.001$, Figure $1 D)$. ROC curves showed that the highest AUC value of FP (0.819) followed by $\mathrm{mFI}$ (0.776) and FDS (0.766) (Figure 1E). Age, sex, and T3-4/ $\mathrm{N}$-adjusted logistic regression analyses showed that frailty was significantly associated with TMT selection in FP (HR 1.63, 95\% CI: $1.20-2.23, \mathrm{P}=0.002), \mathrm{mFI}$ (HR 1.99, 95\% CI: 1.29-3.07, P=0.002), and FDS (HR 1.47, 95\% CI: 1.14 $1.90, \mathrm{P}=0.003)$ (Figure 1F).

\section{Secondary outcomes: the effect of TMT on OS}

Figure 2 shows the unadjusted- and adjusted effect of TMT on OS. The unadjusted Kaplan-Meier curve shows significantly poor OS in the TMT group based on the older age, higher prevalence of comorbidities and frailty (Figure $2 A$ ). To adjust these background difference, we used a propensity score for the TMT group including the high-risk disease $(\mathrm{cT} 3-4 / \mathrm{cN}+)$ and frailty (FP, mFI, and FDS) by a logistic regression analysis. The distribution of propensity score was different between the groups (Figure 2B). The background difference was adjusted by the 

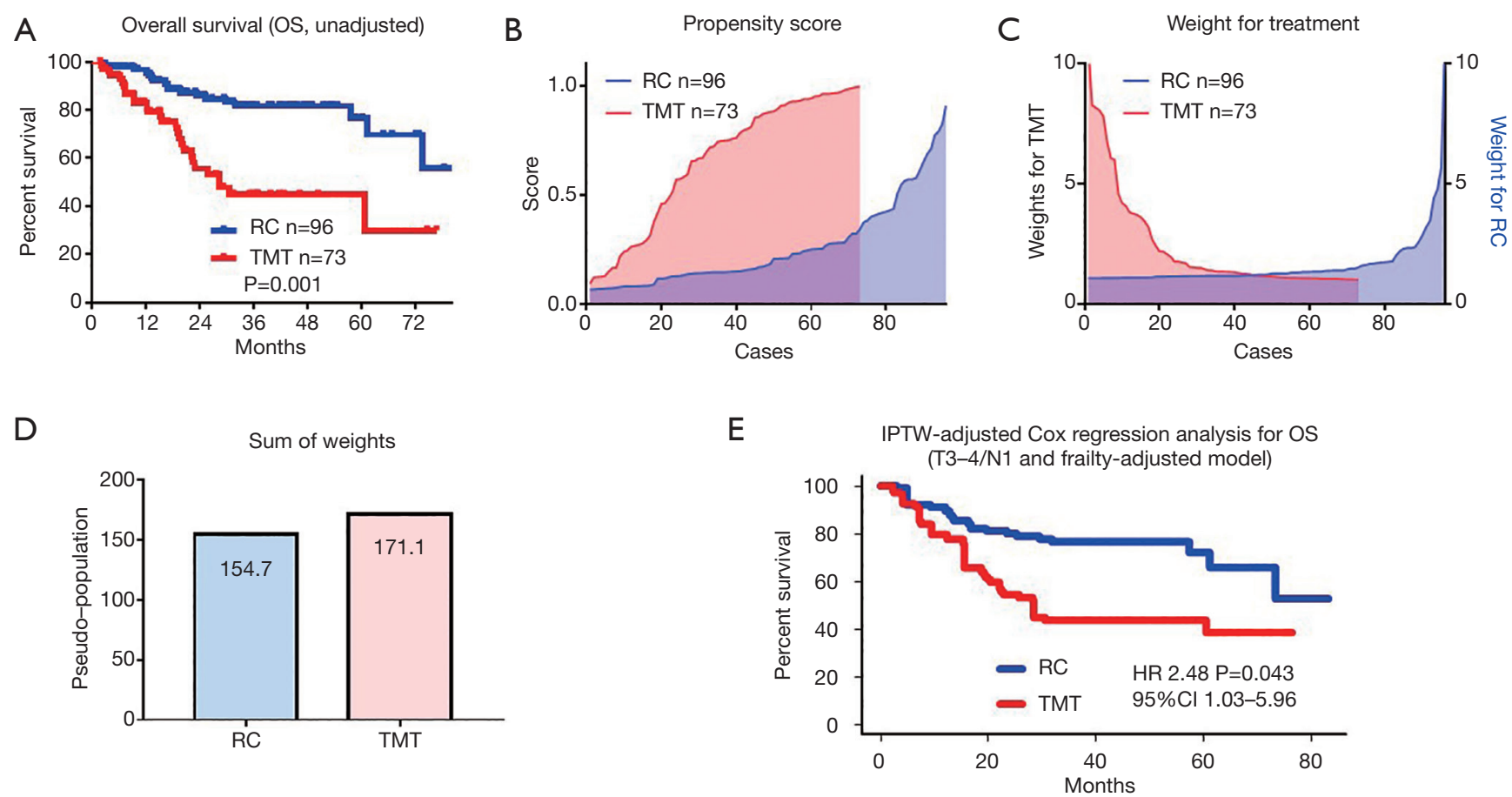

Figure 2 Secondary outcomes: the effect of TMT on overall survival. (A) Overall survival was compared between the RC and TMT groups. Distribution of propensity score (B), inverse probability weights for the TMT group (C), and sum of weights (pseudo-population) (D) were shown. (E) High-risk status (cT3-4/cN+) and frailty-adjusted IPTW-adjusted Cox regression analysis and Kaplan-Meier curve for the effect of frailty on TMT selection was evaluated. RC, radical cystectomy; TMT, trimodal therapy; IPTW, inverse probability of treatment weighting.

inverse-probability of weighting for the TMT group (Figure 2C). The pseudo-population by the IPTW methods was 154.7 and 171.1 in the RC and TMT groups, respectively (Figure 2D). Finally, the IPTW-adjusted Cox regression analysis and the Kaplan-Meier curve showed that the TMT was significantly associated with poor OS (HR 2.48, 95\% CI: 1.03-5.96, $\mathrm{P}=0.043$, Figure 2E).

\section{Exploratory outcomes: the effect of TMT on OS in patient without frailty}

Figure 3 demonstrates unadjusted and IPTW-adjusted OSs in the nonfrail patients who were treated with RC or TMT. A significantly poor OS in the nonfrail patients in the TMT group than that of the RC group was observed in patients with FP nonfrail (Figure 3A), mFI nonfrail (Figure 3B), but not in the patients with FDS nonfrail (Figure 3C). The background-adjusted multivariate Cox regression models using IPTW methods for OS showed that TMT was significantly associated with poor OS in the FP nonfrail (HR
3.40, 95\% CI: 1.36-8.45, $\mathrm{P}=0.002$ ), mFI nonfrail (HR 6.16, 95\% CI: 2.78-13.6, $\mathrm{P}<0.001$ ), and FDS nonfrail (HR 5.22, 95\% CI: 1.84-14.8, P=0.009, Figure 3D).

\section{Discussion}

With the aging population, the development of frailtybased treatment strategies is urgently required for elderly cancer patients $(16,17,19,29)$. However, evidences on the association of frailty and treatment selection for patients with MIBC are limited. Hence, this study retrospectively evaluated the association of frailty with treatment selection (RC or TMT) using three frailty assessment tools. We found that all the three frailty assessment tools showed a significant impact on TMT selection. Of those, the FP $\geq$ 3 might be the most useful for candidate selection of TMT. Moreover, OS was significantly poorer in the TMT group than in the RC group even after the adjustment of baseline disease status, and frailty. OS was found to be significantly different between the RC and TMT groups in nonfrail 

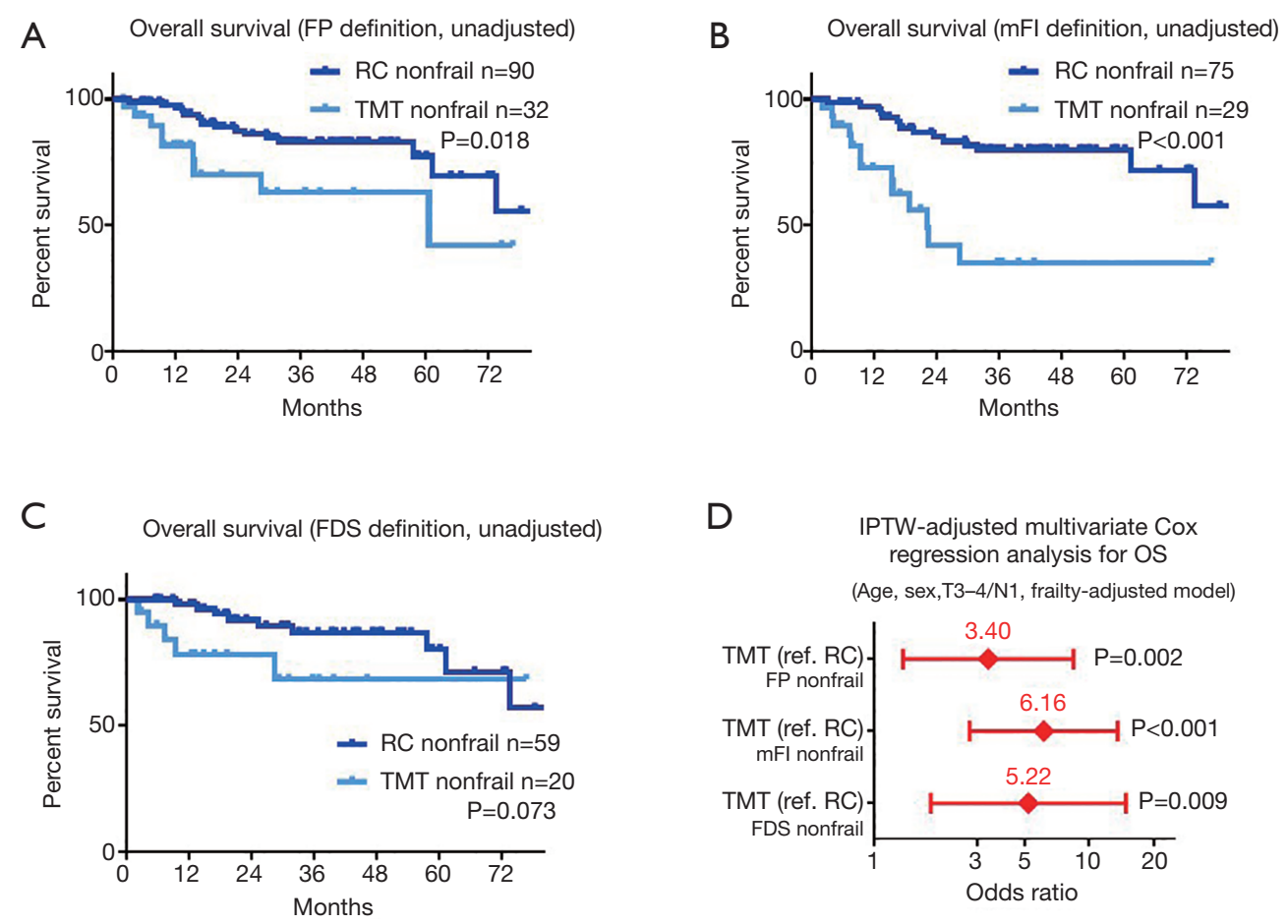

Figure 3 Exploratory outcomes: the effect of TMT on overall survival in patient without frailty. (A,B,C) The unadjusted OS in the nonfrail patients was compared between the RC and TMT groups in the FP, mFI, and FDS. (D) Background (age, sex, T3-4/N+, and frailty)-adjusted Cox regression analyses using IPTW model were performed to evaluate the effect of treatment modality on OS. FP, Fried phenotype; mFI, modified frailty index; FDS, frailty discriminant score; OS, overall survival; RC, radical cystectomy; TMT, trimodal therapy; IPTW, inverse probability of treatment weighting.

patients even after the frailty adjustment. These findings suggest that poor prognosis in patients who received TMT therapy was not only because of frailty but also the effect of treatment modality in real-world practice.

With the increased interest on frailty, majority of the studies focused on the association of frailty with postoperative complications after RC. However, there are few evidences on the use of different frailty assessment tools for a treatment selection in patients with $\operatorname{MIBC}(5,16)$. This is the first study evaluating the difference of frailty between the RC and TMT in patients with MIBC using three different frailty assessment tools. The FP mainly focused on phenotype of frailty, the mFI mainly evaluated comorbidities and disability, and the FDS is a quantitative and comprehensive assessment tool, including the physical, biochemical, and mental status. Despite the difference, all these tools detected the difference in frailty between RC and TMT patients. We found the FP might be the most useful tool for candidate selection of TMT. However, each frailty assessment tool evaluates different dimensional factors of frailty. Future studies are necessary to identify the optimal frailty tool for treatment selection.

Given that there are strong biases for the selection of RC or TMT, the efficacy of TMT on OS in patients with MIBC remains unclear. A recent systematic review suggested that TMT prompts acceptable outcomes and may be an appropriate treatment option in well-selected patients $(9,10,12)$. However, these studies did not account for frailty for patient selection. As our study determined that the prevalence of frailty was significantly different between the groups, frailty is assumed to have some influence on prognosis. We found that OS was significantly worse in the TMT group than in the RC group even in the background- adjusted Cox regression analyses. A higher prevalence of frailty in the TMT group might influence on the limited intensity and efficacy of TMT. Also, OS was significantly worse in the TMT group in nonfrail patients. Although we could not address the reasons, selection biases and unmeasurable confounding factors in the TMT group may have a key role in the poor OS. However, small sample 
size and limited deceased events in this study did not have enough statistical power to detect a clinical implication of frailty on prognosis. A randomized study comparing the effect of treatment modality on prognosis controlling frailty is necessary to elucidate the effect of frailty on treatment modality selection in MIBC patients.

This study had several limitations including a retrospective design, small sample size, and selection bias due to the other unmeasurable confounding factors. In addition, our results may not be representative of those of other countries due to racial and regional differences. Also, the limitation of the IPTW method is the over-weighted effects of minority groups that are far from the center of the patient background. Despite these limitations, this study showed the potential role of frailty in patients with MIBC, highlighting the impact of frailty on treatment selection. As the efficacy and tolerability of immunecheckpoint inhibitors have been reported (30), a novel TMT regimen comprised of TURBT, radiotherapy, and immunecheckpoint inhibitor may improve the oncological outcomes in frail patients with MIBC. Further studies are needed to establish optimal treatment strategies for frail patients with MIBC.

\section{Conclusions}

Frailty was significantly different between the RC and TMT and associated with treatment selection in patients with MIBC.

\section{Acknowledgments}

We thank Yuki Fujita, Yukie Nishizawa, and the entire staff of the Department of Urology in Hirosaki University for their invaluable helps with data collection.

Funding: This study was supported by the Japan Society for the Promotion of Science grant No. 18K09157 (T Yoneyama), 19H05556 (C Ohyama), 19K18595 (H Iwamura), 20K09517 (S Hatakeyama), 20K18082 (Y Kojima), 20K18130 (T Narita) and a research grant from the 31st Japanese Society of Geriatric Urology (S Hatakeyama).

\section{Footnote}

Reporting Checklist: The authors have completed the STROBE reporting checklist. Available at http://dx.doi. org/10.21037/tau-20-1351
Data Sharing Statement: Available at http://dx.doi. org/10.21037/tau-20-1351

Conflicts of Interest: All authors have completed the ICMJE uniform disclosure form (available at http://dx.doi. org/10.21037/tau-20-1351). The authors have no conflicts of interest to declare.

Ethical Statement: The authors are accountable for all aspects of the work in ensuring that questions related to the accuracy or integrity of any part of the work are appropriately investigated and resolved. The study was conducted in accordance with the Declaration of Helsinki (as revised in 2013). The study was approved by the ethics review board of Hirosaki University School of Medicine (NO.: 2014-297) and individual consent for this retrospective analysis was waived.

Open Access Statement: This is an Open Access article distributed in accordance with the Creative Commons Attribution-NonCommercial-NoDerivs 4.0 International License (CC BY-NC-ND 4.0), which permits the noncommercial replication and distribution of the article with the strict proviso that no changes or edits are made and the original work is properly cited (including links to both the formal publication through the relevant DOI and the license). See: https://creativecommons.org/licenses/by-nc-nd/4.0/.

\section{References}

1. RE Hautmann, RC de Petriconi, C Pfeiffer et al. Radical Cystectomy for Urothelial Carcinoma of the Bladder without Neoadjuvant or Adjuvant Therapy: Long-Term Results in 1100 Patients. Eur Urol 2012;61:1039-47.

2. Fujimura T. Current status and future perspective of robot-assisted radical cystectomy for invasive bladder cancer. Int J Urol 2019;26:1033-42.

3. Kimura S, Iwata T, Foerster B, et al. Comparison of perioperative complications and health-related quality of life between robot-assisted and open radical cystectomy: A systematic review and meta-analysis. Int J Urol 2019 ;26:760-74.

4. Sathianathen NJ, Kalapara A, Frydenberg M, et al. Robotic Assisted Radical Cystectomy vs Open Radical Cystectomy: Systematic Review and Meta-Analysis. J Urol 2019;201:715-20.

5. Qu LG, Lawrentschuk N. Orthotopic Neobladder 
Reconstruction: Patient Selection And Perspectives. Res Rep Urol 2019;11:333-41.

6. VanderWalde NA, Chi MT, Hurria A, et al. Treatment of muscle invasive bladder cancer in the elderly: navigating the trade-offs of risk and benefit. World J Urol 2016;34:3-11.

7. Tanaka H, Fukushima H, Kijima T, et al. Feasibility and outcomes of selective tetramodal bladder-preservation therapy in elderly patients with muscle-invasive bladder cancer. Int J Urol 2020;27:236-43.

8. García-Perdomo HA, Montes-Cardona CE, Guacheta M, et al. Muscle-invasive bladder cancer organ-preserving therapy: systematic review and meta-analysis. World J Urol 2018;36:1997-2008.

9. Hamad J, McCloskey H, Milowsky MI, et al. Bladder preservation in muscle-invasive bladder cancer: a comprehensive review. Int Braz J Urol 2020;46:169-84.

10. Kulkarni GS, Hermanns T, Wei Y, et al. Propensity Score Analysis of Radical Cystectomy Versus Bladder-Sparing Trimodal Therapy in the Setting of a Multidisciplinary Bladder Cancer Clinic. J Clin Oncol 2017;35:2299-305.

11. Inamoto T, Ibuki N, Komura K, et al. Can bladder preservation therapy come to the center stage? Int J Urol 2018;25:134-40.

12. Kijima T, Tanaka H, Koga F, et al. Selective tetramodal bladder-preservation therapy, incorporating induction chemoradiotherapy and consolidative partial cystectomy with pelvic lymph node dissection for muscle-invasive bladder cancer: oncological and functional outcomes of 107 patients. BJU Int 2019;124:242-50.

13. Kijima T, Tanaka H, Uehara S, et al. Clinical Outcomes of Patients With Histologic Variants of Urothelial Carcinoma Treated With Selective Tetramodal Bladder-preservation Therapy Incorporating Consolidative Partial Cystectomy. Clin Genitourin Cancer 2020;18:268-273.e2.

14. Sathianathen NJ, Jarosek S, Lawrentschuk N, et al. A Simplified Frailty Index to Predict Outcomes After Radical Cystectomy. Eur Urol Focus 2019;5:658-63.

15. Pearl JA, Patil D, Filson CP, et al. Patient Frailty and Discharge Disposition Following Radical Cystectomy. Clin Genitourin Cancer 2017;15:e615-21.

16. Momota M, Hatakeyama S, Soma O, et al. Geriatric 8 screening of frailty in patients with prostate cancer. Int $\mathrm{J}$ Urol 2020;27:642-8.

17. Okita K, Hatakeyama S, Momota M, et al. Frailty is significantly associated with the type of urinary diversion in patients with muscle-invasive bladder cancer. Int J Urol 2020;27:649-54.

18. Kodama H, Hatakeyama S, Momota M, et al. Effect of frailty and comorbidity on surgical contraindication in patients with localized prostate cancer (FRART-PC Study). Urol Oncol 2021;39:191.e1-191.e8.

19. Ethun CG, Bilen MA, Jani AB, et al. Frailty and cancer: Implications for oncology surgery, medical oncology, and radiation oncology. CA cancer J Clin 2017;67:362-77.

20. Soma O, Hatakeyama S, Okamoto T, et al. Clinical implication of a quantitative frailty assessment tool for prognosis in patients with urological cancers. Oncotarget 2018;9:17396-405.

21. Soma O, Hatakeyama S, Okamoto T, et al. Multicenter prospective study validating the efficacy of a quantitative assessment tool for frailty in patients with urological cancers. Med Oncol 2019;36:88.

22. Iwamoto H, Morizane S, Koie T, et al. Peri-operative efficacy and long-term survival benefit of robotic-assisted radical cystectomy in septuagenarian patients compared with younger patients: a nationwide multi-institutional study in Japan. Int J Clin Oncol 2019;24:1588-95.

23. Okita K, Hatakeyama S, Fujita N, et al. Postoperative weight loss followed by radical cystectomy predicts poor prognosis in patients with muscle-invasive bladder cancer. Med Oncol 2018;36:7.

24. Koie T, Ohyama C, Makiyama K, et al. Utility of robotassisted radical cystectomy with intracorporeal urinary diversion for muscle-invasive bladder cancer. Int J Urol 2019;26:334-40.

25. Burg ML, Clifford TG, Bazargani ST, et al. Frailty as a predictor of complications after radical cystectomy: A prospective study of various preoperative assessments. Urol Oncol 2019;37:40-7.

26. Chappidi MR, Kates M, Patel HD, et al. Frailty as a marker of adverse outcomes in patients with bladder cancer undergoing radical cystectomy. Urol Oncol 2016;34:256.e1-6.

27. Soma O, Hatakeyama S, Imai A, et al. Relationship between frailty and lower urinary tract symptoms among community-dwelling adults. Low Urin Tract Symptoms 2020;12:128-36.

28. Austin PC, Stuart EA. Moving towards best practice when using inverse probability of treatment weighting (IPTW) using the propensity score to estimate causal treatment 
effects in observational studies. Stat Med 2015;34:3661-79.

29. Momota M, Hatakeyama S, Soma O, et al. Frailty is a predictor of moderate to severe pain after robot-assisted

Cite this article as: Iwamura $\mathrm{H}$, Hatakeyama S, Momota M, Kojima Y, Narita T, Okamoto T, Fujita N, Hamano I, Togashi K, Hamaya T, Yoneyama T, Yamamoto H, Yoneyama T, Hashimoto Y, Ohyama C. Relationship of frailty with treatment modality selection in patients with muscle-invasive bladder cancer (FRART-BC study). Transl Androl Urol 2021;10(3):11431151. doi: $10.21037 /$ tau-20-1351 laparoscopic prostatectomy: A case-control study (FRAP study). BJUI Compass 2020;1:100-7.

30. Nakayama T, Kitano S. Immunotherapy for genitourinary tumors. Int J Urol 2019;26:326-33. 